\title{
Imaging of the parathyroid glands in primary hyperparathyroidism
}

\author{
Salvatore Minisola* , Cristiana Cipriani, Daniele Diacinti ${ }^{1}$, Francesco Tartaglia ${ }^{2}$, \\ Alfredo Scillitani ${ }^{3}$, Jessica Pepe and David Scott-Coombes ${ }^{4, *}$ \\ Department of Internal Medicine and Medical Disciplines, ${ }^{1}$ Department of Radiological Sciences, Oncology and \\ Pathology, ${ }^{2}$ Department of Surgical Sciences, "Sapienza" Rome University, Via del Policlinico 155, 00161 Rome, Italy, \\ ${ }^{3}$ Unit of Endocrinology, "Casa Sollievo della Sofferenza" Hospital, San Giovanni Rotondo, Foggia, Italy and \\ ${ }^{4}$ Department of Surgery, University Hospital of Wales, Heath Park, Cardiff, Wales, UK \\ *(S Minisola and D Scott-Coombes are joint senior authors)
}

Correspondence should be addressed to S Minisola or D Scott-Coombes Email

salvatore.minisola@ uniroma1.it or dscottcoombes@ hotmail.com

\begin{abstract}
Primary hyperparathyroidism (PHPT) is one of the most frequent endocrine diseases worldwide. Surgery is the only potentially curable option for patients with this disorder, even though in asymptomatic patients 50 years of age or older without end organ complications, a conservative treatment may be a possible alternative. Bilateral neck exploration under general anaesthesia has been the standard for the definitive treatment. However, significant improvements in preoperative imaging, together with the implementation of rapid parathyroid hormone determination, have determined an increased implementation of focused, minimally invasive surgical approach. Surgeons prefer to have a localization study before an operation (both in the classical scenario and in the minimally invasive procedure). They are not satisfied by having been referred a patient with just a biochemical diagnosis of PHPT. Imaging studies must not be utilized to make the diagnosis of PHPT. They should be obtained to both assist in determining disease etiology and to guide operative procedures together with the nuclear medicine doctor and, most importantly, with the surgeon. On the contrary, apart from minimally invasive procedures in which localization procedures are an obligate choice, some surgeons believe that literature on parathyroidectomy over the past two decades reveals a bias towards localization. Therefore, surgical expertise is more important than the search for abnormal parathyroid glands.
\end{abstract}

\section{Introduction}

Primary hyperparathyroidism (PHPT) is one of the most frequent endocrine diseases worldwide. Surgery is the only potentially curable option for patients with this disorder, even though in asymptomatic patients 50 years of age or older without end organ complications, a conservative treatment may be a possible alternative, according to recent guidelines $(1,2)$. Bilateral neck exploration (BNE) under general anaesthesia has been the standard for the definitive treatment. However, significant improvements in preoperative imaging, together with the implementation of rapid parathyroid hormone (PTH) determination, have determined an increased adoption of focused, minimally invasive surgical approach (3).
(C) 2016 European Society of Endocrinology Printed in Great Britain
Theoretically, preoperative imaging studies should not be used when planning traditional 'open' parathyroid surgery. On the contrary, the minimally invasive procedure assumes accurate preoperative localization of abnormal parathyroid gland(s) (4). Before discussing for or against preoperative localization procedures, we believe it is important to briefly summarize the most commonly techniques utilized.

High-resolution ultrasound (US) represents, together with nuclear medicine imaging, a reliable first-line modality for preoperative localization of a parathyroid lesion. Neck US is currently performed with a highfrequency $(7.5-15 \mathrm{MHz})$ transducer to enhance spatial

Published by Bioscientifica Ltd. 
resolution, thus allowing detection of glands larger than $5 \mathrm{~mm}$ (5). Colour Doppler assessment is a useful integration for the distinction of parathyroid lesions from other cervical masses, thus reducing false positive findings (6). These can also occur in cases of thyroid nodules or concomitant Hashimoto's thyroiditis. The sensitivity of US technique is strongly dependent on the operator's experience, being about $84 \%$ when performed by an experienced doctor (7). Advantages of the US technique include low cost, wide availability, absence of ionizing radiation exposure, together with the possibility of evaluating concomitant thyroid disease (8). Nonetheless, US does not allow detection of small lesions. Furthermore, ectopic glands and the presence of concomitant large thyroid goiter may cause false negative findings (9).

${ }^{99 \mathrm{~m}}$ Technetium $\left({ }^{99 \mathrm{~m}} \mathrm{Tc}\right.$ )-sestamibi (methoxyisobutyl isonitrile (MIBI)), is the agent of choice for the identification of abnormal parathyroid glands by scintigraphy. It is a lipophilic cation entering the over-activated mitochondria rich oxyphil cells. The differential washout rate of this tracer from the thyroid relative to the parathyroid gland allows parathyroid scintigraphy to be performed with this single tracer according to the so-called dual phase procedure. Combining sestamibi with a tracer exclusively taken up by the thyroid tissue (double-tracer technique) permits the subtraction of thyroid imaging, thus avoiding false positive results deriving from the concentrations of sestamibi by solid thyroid nodules. The two techniques have a similar diagnostic performance for localization of single parathyroid adenoma, with a $88-90 \%$ sensitivity. The specificity is over $90 \%$ when using the double tracer technique. Sensitivity and specificity is reported to be even better with the use of early ${ }^{99 \mathrm{~m}} \mathrm{Tc}$ sestamibi-single-photon emission computerized tomography (SPECT) and SPECT/ computed tomography (CT), allowing a 3-D visualization (10). These last procedures should be considered in patients with ectopic parathyroid adenomas or in those who have undergone previous surgery $(11,12)$.

CT (and magnetic resonance imaging (MRI)) is considered a second-line technique useful in cases of ectopic glands or when there is persistence or recurrence of the disease after initial operation. A new 4-D CT technique, combining anatomic and functional information has been recently developed, which takes advantage of the evaluation of enhancement patterns of abnormal parathyroid glands reaching a sensitivity of $85.7 \%$ (13). The use of MRI allows the evaluation of parathyroid anatomy, detection of ectopic glands, in the absence of patient's radiation exposure; reported sensitivity is $80 \%$ with the $1.5 \mathrm{~T}$ magnets (14). Better visualization has recently been obtained with the use of 3.0 T MRI magnet as a valid method for parathyroid lesions identification, particularly in PHPT patients with negative first-line imaging $(15,16)$.

\section{Section 1: Discussion in favour of the argument for routine parathyroid localization for PHPT (Salvatore Minisola, Cristiana Cipriani, Daniele Diacinti, Francesco Tartaglia, Alfredo Scillitani, Jessica Pepe)}

About 30 years ago, when we started to be interested in PHPT (17), the only clinical task was to make the diagnosis. Imaging of the parathyroid glands was an almost unexplored field and patients were immediately referred to the surgeon with expertise in neck operation.

During the last three decades, there has been a flourish of imaging modalities to visualize abnormal parathyroid glands. These are sometimes difficult to compare because of the different settings in which they are employed (i.e. sensitivity of a particular technique is different in the setting of a large parathyroid adenoma vs small adenoma or multiple gland disease (MGD)). This surge of techniques has been mainly fuelled by the need of a precise localization of the abnormal gland(s) in order to perform a minimally invasive parathyroidectomy (PTx). The last could result, among other things, in saving of money, due to shorter time of operation.

In the next section, we argue for a routine preoperative parathyroid localization based on four main arguments.

\section{Surgical expertise}

A number of studies have demonstrated that successful PTX is predominantly dependent on the surgeon's experience rather than the technique employed $(3,18)$. Indeed, both traditional and focused approaches showed excellent outcomes in terms of cure and complications rate depending on experience of both the surgeon and the center (18). Data on long-term follow-up in patients who had undergone parathyroid surgery showed curative rates of $95-98 \%(18,19,20)$. These last data apply to centres with high quality surgery teams and large series, reflecting high expertise in the field (18).

Less data are available from centres with lower parathyroid surgical experience, but collectively suggest that the absence of specific expertise represents the actual 
challenge (18). In this context, few studies evaluated the impact of surgical expertise on parathyroid surgery in terms of number of patients referred to surgery, i.e. surgical volume $(21,22,23)$. This parameter has been found to influence curative rate, operative failure and complications. Historical data from Scandinavia illustrated that $76 \%$ of patients who had undergone PTx in general survey parathyroid surgery were normocalcemic after an average follow-up of 4.4 years, compared with $90 \%$ in specialized surgery centres (21). Additionally, less experienced centres reported higher incidence of persistent PHPT (15\%) and permanent hypoparathyroidism (14\%) (21). Studies in the United States reported persistent PHPT being more common at the low volume centers (87\%) vs the high volume centers (43\%) (22). Mitchell et al. (23) reported higher rates of avoidable parathyroid re-operations in low-volume (78\%) vs high-volume centres $(22 \%)$. These data are of note, particularly considering that re-operative surgery is associated with higher complications rate (22).

The overall data substantially confirm what generally is also observed in clinical practice, i.e. the need for experienced and trained surgery teams as the key point for ensuring successful PTx. PHPT represents a curable disease. However, the availability of experienced parathyroid surgeon is currently the greatest challenge in PHPT management and in predicting patient's outcome (3).

Based on these premises and on the real world evidence of the existence of few centres with very qualified surgical teams, we favour the use of preoperative imaging studies. This is especially important if the operation is carried out by a surgeon with low volume procedures. The availability of pre-surgical localization renders the surgeon more confident and reduces the procedure's length and costs.

\section{Availability of rapid PTH assay}

Rapid PTH measurement has developed in the last decades as a valuable tool in the surgical management of patients undergoing neck exploration for PHPT $(24,25)$. Numerous studies have shown that the intraoperative evaluation of serum PTH (ioPTH) has high accuracy in predicting surgical cure and can function as a guide for surgeon's judgment (25). The short half-life of the hormone in patients with normal renal function enables the use of serum PTH drop after parathyroid resection as an appropriate index of surgical cure $(3,26)$. The absence of serum PTH reduction after surgical removal of the parathyroid gland conversely represents an indication for surgeons to perform additional neck exploration (3). Several cutoff criteria and mathematical model have developed over time to predict the percent change of surgical cure showing positive results, particularly in MGD $(25,27)$. Intra-operative PTH measurement is therefore considered essential, in association with pre-operative imaging studies, for the adoption of minimally invasive techniques (3). Hence, the availability of rapid PTH assay is a key point in any surgery center aimed to perform minimally invasive PTx. In this context, it must be recognized that the use of rapid PTH assays assumes additional costs, related to the need for trained staff, instrumentation and collaboration with local laboratory (25). This point raises some considerations about the need to include experienced laboratories in specialized parathyroid centers performing minimally invasive procedures. As a fact, rapid PTH assay is not available to many surgery centers around the world (3). Statistical data from 2002 have reported that the majority of rapid PTH tests were performed by central laboratories, $23 \%$ by on-site laboratories (i.e. located in the operating room) and $6 \%$ by satellite laboratories (28). The 2014 guidelines for surgical management of PHPT recommended the use of focused procedures in centers able to perform rapid intraoperative PTH measurement as part of the operative protocol (3).

This point again emphasizes that in the real world not all surgical theaters are able to provide 'on site' measurement of ioPTH. As this is a pre-requisite for focused surgery, most surgeons are forced to open cervical explorations. Once more, previous considerations of usefulness of pre-operative imaging for surgeons are therefore valid.

\section{Localization is an obligate choice in patients with persistent or recurrent PHPT}

The goal of localization through imaging techniques in patients with persistent or recurrent PHPT after an unsuccessful operation is to obtain an adequate road map to guide the surgeon, thus achieving a definitive cure and reducing complications. Indeed, reoperation is associated with a potential threefold increase in morbidity and a risk of low curative rate. This is mainly due to distortion and scarring of surgical planes caused by the initial surgery (29).

However, at the present time, the routine use of wellestablished preoperative localization techniques in highvolume centers, resulted in cure rates of a second PTx that are almost the same as those reported in primary 
operations for PHPT (30). Among the conventional noninvasive techniques usually employed to identify parathyroids glands, those performed after a previous neck surgery have different sensitivity compared with the same techniques performed before surgery; more than a single technique is usually performed in the same patient (11). The preferred sequence of imaging continues to evolve and significant institutional variation exists.

As initially described, the first imaging study is usually US, together with nuclear medicine imaging. However, in patients with persistent PHPT, the ${ }^{99 \mathrm{~m}} \mathrm{Tc}$-MIBI sensitivity has been show to decline from $80 \%$, before initial surgery, to $50 \%(12,31)$. If localization is not obtained with US and ${ }^{99 \mathrm{~m}}$ Tc-MIBI scan, additional imaging studies should be performed. For example, a recent study has shown that sestamibi-negative patients who had undergone 4D-CT also had a high rate of surgical concordance with imaging results $(P<0.0001)(32)$. 4D-CT also has the advantage of better identifying ectopic parathyroid glands that are commonly found in conditions of recurrent or persistent PHPT disease. Magnetic resonance has been reported to have a sensitivity of $67 \%$ in patients re-operated (33). If mediastinal parathyroid adenoma is suspected, at least one of these two last imaging techniques is mandatory to assist in the transthoracic operative approach (i.e. median sternotomy or thoracoscopic).

When non-invasive investigations are negative or equivocal, invasive studies such as selective venous sampling and parathyroid arteriography are warranted. In addition, selective venous sampling significantly improves 4D-CT localization of parathyroid adenomas in those patients with negative ${ }^{99 \mathrm{~m}} \mathrm{Tc}-\mathrm{MIBI}$ and US, increasing the sensitivity from $50 \%$ to $95 \%$ $(P=0.004)(34)$.

Therefore, judicious use of localization studies in patients unsuccessfully operated on for PHPT allows the same success rate of initial operations, with a minimized risk of recurrent laryngeal nerve palsy and with a decreased prevalence of permanent hypoparathyroidism. Moreover, other complications such as wound infection, postoperative bleeding, and pneumonia were shown to be significantly lower in a group that underwent repeated PTx between 1998 and 2008 when more sophisticated diagnostic tools were available compared to a group that underwent re-operation between 1987 and 1997 $(P<0.001)(30)$.

These data strongly indicate that, localization studies in PHPT patients with persistent or recurrent disease are an obligate choice before performing a second operation.

\section{Costs}

Another important point to favor the utilization of imaging studies, before surgical procedures for PHPT, is the potential to save money. There are few detailed data on this topic. However, an exhaustive study carried out by Pata et al. (35) showed, for example, that the utilization of SPECT/CT ensures better focus for the surgical exploration, shortens surgical times and eventually cut costs when used for localization of parathyroid surgery.

\section{Conclusions}

We believe that in 2015, the majority of surgeons prefer to have a localization study before operation and are not satisfied by having been referred a patient with just a biochemical diagnosis of PHPT. This might also have some legal implications in case of surgery failure. The crucial point is that imaging studies must by no means be utilized to make the diagnosis of PHPT. They should be obtained to both assist in determining disease etiology and to direct operative procedures. As such, they should be ordered and discussed in conjunction with the nuclear medicine doctor and most importantly with the surgeon. Finally, more complex cases, that is, patients with MGD, negative preoperative imaging studies, persistent and recurrent disease should be managed in experienced tertiary centers with a volume of at least 50 PTx a year (22).

The time has come for optimally combining the best that technology has to offer in this scenario with the best surgical expertise, for ensuring maximum benefit to patients. We therefore propose the algorithm illustrated in Fig. 1, as a wise approach balancing the needs and expectations of surgeons and taking into account a pragmatic real world situation.

\section{Section 2: Discussion opposing the argument against routine parathyroid localization for PHPT (David Scott-Coombes)}

The traditional approach for PTx in patients with PHPT is a BNE and the desired outcomes are: i) to achieve lasting normocalcemia, ii) with minimum morbidity, iii) a short length of stay, and iv) with minimal pain and minimal scarring.

BNE had a 95\% normocalcemia rate in the best units (36) but undoubtedly there is a publication bias. BNE can be undertaken with minimum morbidity, minimal discomfort and an excellent cosmetic outcome in a $23 \mathrm{~h}: 59 \mathrm{~m}$ admission. The most common reasons why 


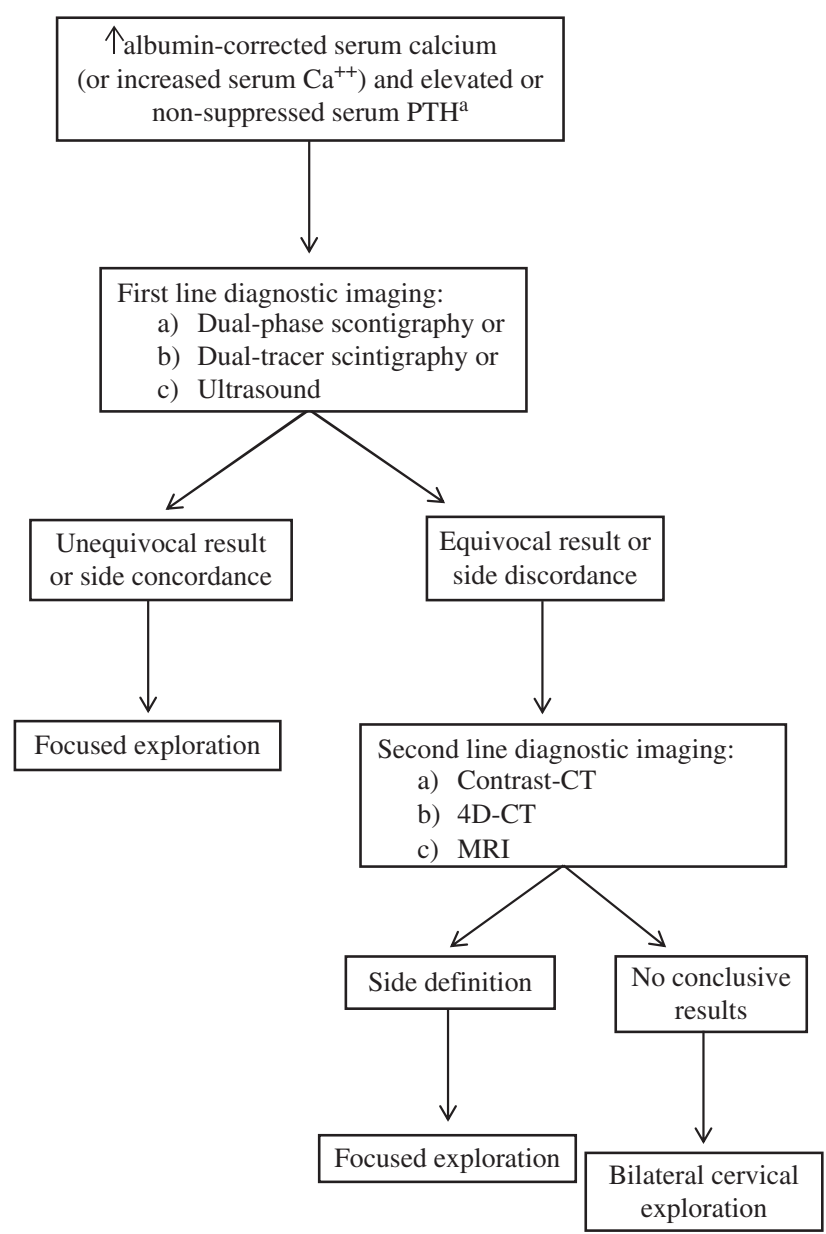

\section{Figure 1}

Initial diagnostic imaging approach in patients with primary hyperparathyroidism, without previous parathyroid surgery.

${ }^{a}$ Exclude familial hypocalciuric hypercalcemia.

surgeons fail (i.e. $<100 \%$ normocalcemia rate) are ectopia (53\%) and MGD (37\%) (37). However most 'ectopic' glands were in well-recognized locations including the paraesophageal space and thymus. In a recent series of remedial parathyroid surgery for first-time failures, 38\% of glands were ectopic, of which most (68\%) were in 'typical' locations (38). These outcomes highlight the importance of surgeon experience. There is strong evidence that better outcomes are achieved in units who undertake more than 50 cases per year (22).

Minimal invasive PTx (MIP) has replaced BNE as the procedure of choice when a solitary adenoma can be localized pre-operatively (19). The benefits of MIP include shorter operating time, less pain, less scarring, shorter length-of-stay and less cost. MIP is successful in expert centres. When failures occur, MGD accounts for the majority because ectopia would be predicted by preoperative localization. For example, in a series of 1000 MIPs in Sydney, failures (1.7\%) were due to missed double adenoma (59\%) and missed four-gland hyperplasia (18\%) (39).

But MIP is only feasible when localization is successful. How successful is localization? The sensitivity for US ranges between 60 and $75 \%(40,41)$ and is very operator dependant. The sensitivity is higher for MIBI scintigraphy. A wide variety of sensitivities are reported (54-96\%) (4), with an overall sensitivity around 85\% (41). Sensitivity can be ameliorated by the use of dual tracer subtraction method (12). 4D-CT technique has reports of a sensitivity around $90 \%$ (42).

What is clear is that no localization technique is $100 \%$ reliable. This unreliability deserves further consideration. Localization fails for two important reasons.

\section{Localization is unreliable in patients with MGD}

While a meta-analysis of over 20000 studies reports an overall sensitivity of $88 \%$ for detection of a single adenoma (43), this sensitivity collapses to $45 \%$ in patients with four-gland hyperplasia and 30\% for double adenoma. US fares even worse: 35\% for four-gland hyperplasia and a mere $16 \%$ for double adenoma.

\section{Localization is unreliable for small parathyroid tumors}

Several papers have demonstrated that localization is less successful for smaller tumors $(9,44,45)$. Gland weight below $600 \mathrm{mg}$ is associated with false negative results $(9,46)$.

Logically, identification of a smaller adenoma is going to be more difficult for the surgeon compared with finding a larger gland (eutopic or ectopic). Remembering that the most common reasons to fail are failure to identify a tumor and failure to recognise MGD, it is disconcerting that the reliability of pre-operative localization falls away when tumors are smaller and multiple. In other words, when localization is most needed, it is least reliable.

A consequence of pre-operative localization is the emergence of the image-negative patient. This is reported to occur in $12-18 \%$ of cases $(47,48)$ and is a portent of both smaller tumors (49) and MGD, which accounts for one-third of cases $(47,48)$. The Scandinavian Quality Register for Thyroid and Parathyroid Surgery (27 departments and 3158 patients) reported image-negative 
patients to occur $17 \%$ of the time (50). In this series, MGD accounted for $22 \%$ of cases and the median weight of excised tissue was only $350 \mathrm{mg}$. Of concern, the rate of negative explorations in this group was $13 \%$ and the rate of persistent hyperparathyroidism was a worrisomely high $18 \%$. This study highlights the increased complexity of the negative-image group and also raises questions about the role of centralization to high volume centers for this group of patients.

\section{Cost}

In a European randomized trial comparing unilateral neck exploration (UNE) with BNE, there was no difference in cost (51). However, most studies comparing the cost benefit between BNE and MIP largely support the value of MIP $(52,53)$. Duration of time in theater (at $15 \mathrm{~min}$ intervals) has a significant influence on the costs in the USA (54). There is also an assumption that all patients undergoing MIP are not admitted, which is not always the case in UK practice (depending upon the time of day that surgery occurs).

\section{Radiation exposure}

The average annual UK dose of natural background radiation is $2.2 \mathrm{mSv}$. A chest X-ray has a typical dose of $0.02 \mathrm{mSv}$. The average radiation doses in parathyroid imaging are as follows: MIBI $(3.33 \mathrm{mSv})$; dynamic CT $(5.56 \mathrm{mSv})$, SPECT-MIBI $(7.8 \mathrm{mSv})$ (55). However, the greatest radiation dose is $4 \mathrm{D}-\mathrm{CT}(10.4 \mathrm{mSv})$, which is the equivalent of 520 chest-X-rays (56). Such a huge exposure of radiation to the thyroid has led to recommendations that $4 \mathrm{D}$-CT should be used judiciously in young patients.

While MIP is faster than BNE, the benefit is measured in minutes. Day case (outpatient) surgery is not only determined by the surgical approach but influenced by the culture of the National Health Services and the geography of the referral base. While there is an increased risk of recurrent laryngeal nerve injury in BNE, this risk is theoretical and should be close to zero when undertaken by an experienced surgeon. While much is made of the cosmetic benefit of MIP, BNE is undertaken with a $4-5 \mathrm{~cm}$ collar incision with excellent outcomes. In other words, the benefits of MIP over BNE are marginal. But the practice of localization has unwelcome consequences.

Localization is most successful for larger solitary adenomas, which would rarely be a challenge to an experienced surgeon without localization. Therefore localization leads to 'cherry picking' of the easiest cases. As a consequence, BNE is less frequently undertaken and risks deskilling surgeons-in-training.

Another consequence of localization is the emergence of the image-negative patient. MGD and smaller glands are inevitable in this group and there is already evidence that this leads to poorer surgical outcomes outside high volume centres. As PHPT is diagnosed earlier the proportion of image-negative patients is set to rise (57).

First-time PTx for PHPT is an operation that has evolved from one that required no imaging to one that is now dependent on imaging. In 1986, the interventional radiologist Doppman (58) said 'in my opinion the only localizing study indicated in a patient with untreated PHPT is to localize an experienced surgeon' as a reflection of the (in)accuracy of localization studies and the initial attitudes towards them at that time. Any review of the literature on PTx over the past two decades reveals a bias towards localization. The time has come for the focus to shift away from technology and back towards surgical expertise.

Declaration of interest

The authors declare that there is no conflict of interest that could be perceived as prejudicing the impartiality of the research reported.

\section{Funding}

This research did not receive any specific grant from any funding agency in the public, commercial or not-for-profit sector.

\section{References}

1 Bilezikian JP, Brandi ML, Eastell R, Silverberg SJ, Udelsman R, Marcocci C \& Potts JT Jr. Guidelines for the management of asymptomatic primary hyperparathyroidism: summary statement from the Fourth International Workshop. Journal of Clinical Endocrinology and Metabolism 201499 3561-3569. (doi:10.1210/jc.2014-1413)

2 Silverberg SJ, Clarke BL, Peacock M, Bandeira F, Boutroy S, Cusano NE, Dempster D, Lewiecki EM, Liu JM, Minisola S et al. Current issues in the presentation of asymptomatic primary hyperparathyroidism: proceedings of the Fourth International Workshop. Journal of Clinical Endocrinology and Metabolism 201499 3580-3594. (doi:10.1210/ jc.2014-1415)

3 Udelsman R, Åkerström G, Biagini C, Duh QY, Miccoli P, Niederle B \& Tonelli F. The surgical management of asymptomatic primary hyperparathyroidism: proceedings of the Fourth International Workshop. Journal of Clinical Endocrinology and Metabolism 201499 3595-3606. (doi:10.1210/jc.2014-2000)

4 Kunstman JW, Kirsch JD, Mahajan A, Udelsman R, Kunstman JW, Kirsch JD, Mahajan A \& Udelsman R. Clinical Review: Parathyroid localization and implications for clinical management. Journal of 
Clinical Endocrinology and Metabolism 201398 902-912. (doi:10.1210/ jc.2012-3168)

5 Kamaya A, Quon A \& Jeffrey RB. Sonography of the abnormal parathyroid gland. Ultrasound Quarterly 200622 253-262. (doi:10.1097) 01.ruq.0000237260.33509.4f)

6 Devcic Z, Jeffrey RB, Kamaya A \& Desser TS. The elusive parathyroid adenoma: techniques for detection. Ultrasound Quarterly 201329 179-187. (doi:10.1097/RUQ.0b013e3182a1ba6f)

7 Vitetta GM, Neri P, Chiecchio A, Carriero A, Cirillo S, Mussetto AB \& Codegone A. Role of ultrasonography in the management of patients with primary hyperparathyroidism: retrospective comparison with technetium-99m sestamibi scintigraphy. Journal of Ultrasound 201417 1-12. (doi:10.1007/s40477-014-0067-8)

8 Morita SY, Somervell H, Umbricht CB, Dackiw AP \& Zeiger MA. Evaluation for concomitant thyroid nodules and primary hyperparathyroidism in patients undergoing parathyroidectomy or thyroidectomy. Surgery 2008144 862-866. (doi:10.1016/j.surg.2008. 07.029)

9 Berber E, Parikh RT, Ballem N, Garner CN, Milas M \& Siperstein AE. Factors contributing to negative parathyroid localization: an analysis of 1000 patients. Surgery 2008144 74-79. (doi:10.1016/j.surg.2008.03. 019)

10 Guerin C, Lowery A, Gabriel S, Castinetti F, Philippon M, VaillantLombard J, Loundou A, Henry JF, Sebag F \& Taïeb D. Preoperative imaging for focused parathyroidectomy: making a good strategy even better. European Journal of Endocrinology/European Federation of Endocrine Societies 2015172 519-526. (doi:10.1530/EJE-14-0964)

11 Mariani G, Mazzeo S, Rubello D \& Bartolozzi C. Preoperative localization of abnormal parathyroid glands. In Parathyroids, 3rd edn, ch 35, pp 499-518. Ed JP Bilezikian. San Diego: Academic Press, 2015.

12 Hindié E, Zanotti-Fregonara P, Tabarin A, Rubello D, Morelec I, Wagner T, Henry JF \& Taïeb D. The role of radionuclide imaging in the surgical management of primary hyperparathyroidism. Journal of Nuclear Medicine 201556 737-744.

13 Starker LF, Mahajan A, Björklund P, Sze G, Udelsman R \& Carling T. 4D parathyroid CT as the initial localization study for patients with de novo primary hyperparathyroidism. Annals of Surgical Oncology 201118 1723-1728. (doi:10.1245/s10434-010-1507-0)

14 Lopez Hänninen E, Vogl TJ, Steinmüller T, Ricke J, Neuhaus P \& Felix R. Preoperative contrast-enhanced MRI of the parathyroid glands in hyperparathyroidism. Investigative Radiology 200035 426-430.

15 Grayev AM, Gentry LR, Hartman MJ, Chen H, Perlman SB \& Reeder SB. Presurgical localization of parathyroid adenomas with magnetic resonance imaging at $3.0 \mathrm{~T}$ : an adjunct method to supplement traditional imaging. Annals of Surgical Oncology 201219 981-989. (doi:10.1245/s10434-011-2046-z)

16 Sacconi B, Argirò R, Diacinti D, Iannarelli A, Bezzi M, Cipriani C, Pisani D, Cipolla V, De Felice C, Minisola S et al. MR appearance of parathyroid adenomas at $3 \mathrm{~T}$ in patients with primary hyperparathyroidism: what radiologists need to know for pre-operative localization. European Radiology 2015. In Press. (doi:10.1007/s00330-015-3854-5)

17 Minisola S, Scarnecchia L, Scarda A, Bigi F, Tabolli S, Valtorta C \& Mazzuoli G. Serum osteocalcin in primary hyperparathyroidism: short-term effect of surgery. Mineral and Electrolyte Metabolism 198814 201-207.

18 Callender GG, Carling T \& Udelmsn R. In Parathyroids, 3rd edn, ch 36, pp 519-532. Ed JP Bilezikian. San Diego: Academic Press, 2015.

19 Udelsman R, Lin Z \& Donovan P. The superiority of minimally invasive parathyroidectomy based on 1650 consecutive patients with primary hyperparathyroidism. Annals of Surgery 2011253 585-591. (doi:10.1097/SLA.0b013e318208fed9)

20 Westerdahl J \& Bergenfelz A. Unilateral versus bilateral neck exploration for primary hyperparathyroidism: five-year follow-up of a randomized controlled trial. Annals of Surgery 2007246 976-980. (doi:10.1097/SLA.0b013e31815c3ffd)
21 Malmaeus J, Granberg PO, Halvorsen J, Akerström G \& Johansson H. Parathyroid surgery in Scandinavia. Acta Chirurgica Scandinavica 1988 154 409-413.

22 Chen H, Wang TS, Yen TW, Doffek K, Krzywda E, Schaefer S, Sippel RS \& Wilson SD. Operative failures after parathyroidectomy for hyperparathyroidism: the influence of surgical volume. Annals of Surgery 2010 252 691-695.

23 Mitchell J, Milas M, Barbosa G, Sutton J, Berber E \& Siperstein A. Avoidable reoperations for thyroid and parathyroid surgery: effect of hospital volume. Surgery 2008144 899-906. (doi:10.1016/j.surg.2008. 07.022)

24 Nussbaum SR, Thomspon AR, Hutcheson BA, Gaz RD \& Wang C. Intraoperative measurement of parathyroid hormone in the surgical management of hyperparathyroidism. Surgery 1988104 1121-1127.

25 Sokoll LJ, Wians FH Jr \& Remaley AT. Rapid intraoperative immunoassay of parathyroid hormone and other hormones: a new paradigm for point-of-care testing. Clinical Chemistry 200450 1126-1133. (doi:10.1373/clinchem.2003.030817)

26 Minisola S, Scarnecchia L, Romagnoli E, Carnevale V, Pacitti MT, Scarda A, Rosso R \& Mazzuoli GF. Conventional and new diagnostic applications of a two-site immunochemiluminometric assay for parathyroid hormone. Journal of Endocrinological Investigation 1992 15 483-489. (doi:10.1007/BF03348784)

27 Udelsman R, Donovan P \& Shaw C. Cure predictability during parathyroidectomy. World Journal of Surgery 201438 525-533. (doi:10.1007/s00268-013-2327-8)

28 Hortin GL \& Carter AB. Intraoperative parathyroid hormone testing: survey of testing program characteristics. Archives of Pathology \& Laboratory Medicine 2002126 1045-1049.

29 Hasse C, Sitter H, Brune M, Wollenteit I, Nies C \& Rothmund M. Quality of life and patient satisfaction after reoperation for primary hyperparathyroidism: analysis of long-term results. World Journal of Surgery 200226 1029-1036. (doi:10.1007/s00268-002-6664-2)

30 Karakas E, Müller HH, Schlosshauer T, Rothmund M \& Bartsch DK. Reoperations for primary hyperparathyroidism - improvement of outcome over two decades. Langenbeck's Archives of Surgery/Deutsche Gesellschaft für Chirurgie 2013398 99-106.

31 Witteveen JE, Kievit J, Stokkel MP, Morreau H, Romijn JA \& Hamdy NA. Limitations of Tc99m-MIBI-SPECT imaging scans in persistent primary hyperparathyroidism. World Journal of Surgery 201135 128-139. (doi:10.1007/s00268-010-0818-4)

32 Cham S, Sepahdari AR, Hall KE, Yeh MW \& Harari A. Dynamic Parathyroid Computed tomography (4DCT) facilitates reoperative parathyroidectomy and enables cure of missed hyperplasia. Annals of Surgical Oncology 201522 3537-3542. (doi:10.1245/s10434-014-4331-0)

33 Yen TW, Wang TS, Doffek KM, Krzywda EA \& Wilson SD. Reoperative parathyroidectomy: an algorithm for imaging and monitoring of intraoperative parathyroid hormone levels that results in a successful focused approach. Surgery 2008144 611-619. (doi:10.1016/j.surg.2008. 06.017)

34 Ginsburg M, Christoforidis GA, Zivin SP, Obara P, Wroblewski K, Angelos P, Grogan RH \& Kaplan EL. Adenoma localization for recurrent or persistent primary hyperparathyroidism using dynamic fourdimensional CT and venous sampling. Journal of Vascular and Interventional Radiology 201526 79-86. (doi:10.1016/j.jvir.2014.09.019)

35 Pata G, Casella C, Magri GC, Lucchini S, Panarotto MB, Crea N, Giubbini R \& Salerni B. Financial and clinical implications of lowenergy CT combined with 99m Technetium-sestamibi SPECT for primary hyperparathyroidism. Annals of Surgical Oncology 201118 2555-2563. (doi:10.1245/s10434-011-1641-3)

36 Auguste LJ, Attie JN \& Schnaap D. Initial failure of surgical exploration in patients with primary hyperparathyroidism. American Journal of Surgery 1990160 333-336. (doi:10.1016/S0002-9610(05)80536-7)

37 Shen W, Düren M, Morita E, Higgins C, Duh QY, Siperstein AE \& Clark $\mathrm{OH}$. Reoperation for persistent or recurrent primary 
hyperparathyroidism. Archives of Surgery 1996131 861-867; discussion 867-869. (doi:10.1001/archsurg.1996.01430200071013)

38 Udelsman R \& Donovan PI. Remedial parathyroid surgery: changing trends in 130 consecutive cases. Annals of Surgery 2006244 471-479.

39 Suliburk JW, Sywak MS, Sidhu SB \& Delbridge LW. 1000 minimally invasive parathyroidectomies without intra-operative parathyroid hormone measurement: lessons learned. ANZ Journal of Surgery 201181 362-365. (doi:10.1111/j.1445-2197.2010.05488.x)

40 Richards ML \& Grant CS. Current applications of the intraoperative parathyroid hormone assay in parathyroid surgery. American Surgeon 200773 311-317.

41 Vazquez BJ \& Richards ML. Imaging of the thyroid and parathyroid glands. Surgical Clinics of North America 201191 15-32. (doi:10.1016/ j.suc.2010.10.015)

42 Mortenson MM, Evans DB, Lee JE, Hunter GJ, Shellingerhout D, Vu T, Edeiken BS, Feng L \& Perrier ND. Parathyroid exploration in the reoperative neck: improved preoperative localization with $4 \mathrm{D}$-computed tomography. Journal of the American College of Surgeons 2008206 888-895; discussion 895-896. (doi:10.1016/j.jamcollsurg.2007.12.044)

43 Ruda JM, Hollenbeak CS \& Stack BC Jr. A systematic review of the diagnosis and treatment of primary hyperparathyroidism from 1995 to 2003. Otolaryngology-Head and Neck Surgery 2005132 359-372.

44 Lee VS, Wilkinson RH Jr, Leight GS Jr, Coogan AC \& Coleman RE. Hyperparathyroidism in high-risk surgical patients: evaluation with double-phase technetium-99m sestamibi imaging. Radiology 1995197 627-633. (doi:10.1148/radiology.197.3.7480730)

45 Erbil Y, Barbaros U, Yanik BT, Salmaslioğlu A, Tunaci M, Adalet I, Bozbora A \& Ozarmağan S. Impact of gland morphology and concomitant thyroid nodules on preoperative localization of parathyroid adenomas. Laryngoscope 2006116 580-585. (doi:10.1097/01. MLG.0000203411.53666.AD)

46 Nichols KJ, Tomas MB, Tronco GG, Rini JN, Kunjummen BD, Heller KS, Sznyter LA \& Palestro CJ. Preoperative parathyroid scintigraphic lesion localization: accuracy of various types of readings. Radiology 2008248 221-232. (doi:10.1148/radiol.2481071066)

47 Sebag F, Hubbard JG, Maweja S, Misso C, Tardivet L \& Henry JF. Negative preoperative localization studies are highly predictive of multiglandular disease in sporadic primary hyperparathyroidism. Surgery 2003134 1038-1341; discussion 1041-1042. (doi:10.1016/ j.surg.2003.07.021)

48 Sugg SL, Hubbard JG, Maweja S, Misso C, Tardivet L \& Henry JF. Detection of multiple gland primary hyperparathyroidism in the era of minimally invasive parathyroidectomy. Surgery 2004136 1303-1309. (doi:10.1016/j.surg.2004.06.062)
49 Goldstein RE, Carter WM II, Fleming M, Bumpous J, Lentsch E, Rice M \& Flynn M. Unilateral cervical surgical exploration aided by intraoperative parathyroid hormone monitoring in patients with primary hyperparathyroidism and equivocal sestamibi scan results. Archives of Surgery 2006141 552-557; discussion 557-559. (doi:10.1001/archsurg. 141.6.552)

50 Bergenfelz AO, Wallin G, Jansson S, Eriksson H, Mårtensson H, Christiansen P \& Reihnér E. Results of surgery for sporadic primary hyperparathyroidism in patients with preoperatively negative sestamibi scintigraphy and ultrasound. Langenbeck's Archives of Surgery/Deutsche Gesellschaft für Chirurgie 2011396 83-90.

51 Bergenfelz A, Lindblom P, Tibblin S \& Westerdahl J. Unilateral versus bilateral neck exploration for primary hyperparathyroidism: a prospective randomized controlled trial. Annals of Surgery 2002236 543-551. (doi:10.1097/00000658-200211000-00001)

52 Fahy BN, Bold RJ, Beckett L \& Schneider PD. Modern parathyroid surgery: a cost-benefit analysis of localizing strategies. Archives of Surgery 2002137 917-922; discussion 922-933. (doi:10.1001/archsurg. 137.8.917)

53 Zanocco K, Heller M \& Sturgeon C. Cost-effectiveness of parathyroidectomy for primary hyperparathyroidism. Endocrine Practice 201117 (Suppl 1) 69-74. (doi:10.4158/EP10311.RA)

54 Lubitz CC, Stephen AE, Hodin RA \& Pandharipande P. Preoperative localization strategies for primary hyperparathyroidism: an economic analysis. Annals of Surgical Oncology 201219 4202-4209. (doi:10.1245/ s10434-012-2512-2)

55 Madorin CA, Owen R, Coakley B, Lowe H, Nam KH, Weber K, Kushnir L, Rios J, Genden E, Pawha PS et al. Comparison of radiation exposure and cost between dynamic computed tomography and sestamibi scintigraphy for preoperative localization of parathyroid lesions. JAMA Surgery 2013148 500-503. (doi:10.1001/ jamasurg.2013.57)

56 Mahajan A, Starker LF, Ghita M, Udelsman R, Brink JA \& Carling T. Parathyroid four-dimensional computed tomography: evaluation of radiation dose exposure during preoperative localization of parathyroid tumors in primary hyperparathyroidism. World Journal of Surgery 2012 36 1335-1339. (doi:10.1007/s00268-011-1365-3)

57 Sitges-Serra A, Rosa P, Valero M, Membrilla E \& Sancho JJ. Surgery for sporadic primary hyperparathyroidism: controversies and evidencebased approach. Langenbeck's Archives of Surgery/Deutsche Gesellschaft für Chirurgie 2008393 239-244.

58 Doppman JL. Reoperative parathyroid surgery; localization procedures. Progress in Surgery 198618 117-132.

Received 7 June 2015

Revised version received 18 August 2015

Accepted 3 September 2015 